\title{
Vacuum Polarization and the Spontaneous Loss of Charge by Black Holes
}

\author{
G. W. Gibbons \\ University of Cambridge, D.A.M.T.P., Cambridge CB3 9EW, U.K.
}

Received June 16, 1974

\begin{abstract}
The spontaneous loss of charge by black holes due to particle emission is discussed. For large black holes (more massive than $10^{17} \mathrm{~g}$ ) the process is shown to be governed by a Schwinger type formula. For smaller black holes the method of calculating the process is described and asymptotic forms for scattering and superradiant coefficients given.
\end{abstract}

\section{Introduction}

The purpose of this paper is to investigate under what circumstances a black hole immersed in a vacuum may possess charge and the mechanism whereby it loses by the spontaneous production of pairs of oppositely charged particles. The motivation for the work is both conceptual - to understand particle production processes in strong gravitational fields- and astrophysical. The astrophysical interest is not so much in black holes of solar mass or above which may have formed from the gravitational collapse of stars, star clusters or galaxies as in black holes of small mass which Hawking [1] has suggested may have formed in the early universe. If these are to be easily detectable than it will be by any charge that they possess, since then they would show up in bubble chamber tracks and possibly using etched track techniques etc.

It is clear that whether or not they may possess charge is crucial to any consideration of searching for such objects. We shall not discuss the astrophysics of small black holes further in this paper but merely note that the question of large black holes is somewhat simpler to answer. One would not normally expect large excesses of charge to occur in the universe and indeed an object of mass $M$ and charge $Q$ will not gravitationally accrete particles of mass $m$, charge $e$, if the inequality

$$
e Q>M m
$$

holds unless the particles are projected at the object with some initial velocity. (Here we are using, as we shall throughout this paper unless the contrary is stated "natural units", n.u. in which $\hbar=c=G=k=1$. The electrical units are unrationalized. Since $m / e \approx 10^{-21}$ for electrons we see that a large black hole will naturally acquire hardly any charge and will probably lose it by accreting particles of opposite sign rather quickly. It will turn out that this implies that the amount of activity due to pair formation will be quite negligible. 
When this work was embarked upon it was rather expected that the situation with respect to quantum processes for non rotating black holes would be as follows.

It is easily seen from a consideration of the charged particle orbits in the Reisner-Nordstrom solution or using the "first law of black hole mechanics" [2] that provided

$$
\frac{\varrho Q}{r_{+}}>m
$$

it is energetically favourable for a pair of oppositely charged particles to form in the vicinity of the hole, for one to fall into the hole and the other to escape to infinity. $r_{+}$is the radius of the event horizon and $\frac{Q}{r_{+}}$is the electric potential at the event horizon.

Criterion (1.2) is necessary for both the existence of a "generalized ergosphere" to exist in the Reisner-Nordstrom - ie a region where the exist orbits with negative total energy cf. [3] and for "superradiant" scattering of waves obeying the charged Klein-Gordon equation. This means that on the level of classical particles a Penrose process [4] is possible and on the level of waves the corresponding phenomenon occurs the so called "Klein Paradox" [5]. These are the complementary aspects of the "wave particle duality" a unified account of which is obtained by an appeal to quantum field theory. Since Eq. (1.2) does not - when conventional units are restored contain Planck's constant one seeks a criterion which governs the rate of this essentially quantum process. Such a quantity is the so called "critical field strength", and at least for large black holes such that

$$
M \gg \frac{1}{m}
$$

that is the Schwarzchild radius is much larger than the Compton wavelength of the particle we might hope to apply Schwinger's well known result [6] for the rate of particles created by a uniform electric field. Thus we expect the rate to be very slow unless

$$
\frac{e Q}{r_{+}^{2}}>\frac{m^{2}}{e}
$$

and to rapid otherwise their being an exponential dependence of the form

$$
\exp -\frac{\pi m^{2} r_{+}^{2}}{e Q}
$$

If we plot on a diagram of $Q$ against $M$ for black holes the lines $Q=e ; Q=M$, $e Q=M m$, and $e Q=M^{2} m^{2}$ see Fig. 1 we come to the following conclusions [7] (since $M \leqq r_{+} \leqq 2 M$ ).

1) To possess even one electron charge for a reasonable length of time the hold must have a mass greater than $\frac{e^{2}}{m} \approx 10^{15} \mathrm{~g}$. 


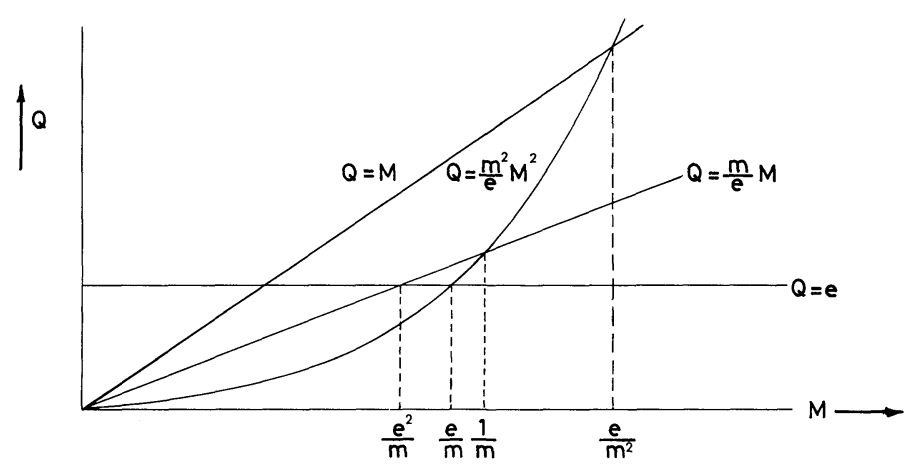

Fig. 1. A diagram of $Q$ against $M$ for black holes (taken for simplicity as non rotating) all black holes lie below the lines $Q=M$ (otherwise there would be no event horizon) and above $Q=e$ (since charge is quantized). Above $Q=\frac{m}{e} M$ it is energetically favourable to form pairs. Above $Q=\frac{m^{2} M^{2}}{e}$ this is a rapid process. To make a sensible diagram the magnitude of $\frac{m}{e}$ is much greater than its physical value

2) To possess a charge at all significant with its mass a hole must be more massive than $\frac{e}{m^{2}} \approx 10^{5} M_{\odot}$.

Where we have used the mass and charge of the electron since it is the lightest and hence most easily emitted particle.

However it has now become clear from the remarkable work of Hawking that these results which will remain true for large black holes in the sense that $M \gg \frac{1}{m}$ they require substantial modification below that limit $[8,9]$.

In addition to the emission in superradiant modes (which has also been discussed by Starobinsky [20] and Unruh [11] in the different but closely related context of rotating holes) he has discovered the time dependence of a general gravitational collapse is such as to cause even a neutral, non rotating black hole to emit particles of all kinds as if it were a body with a temperature $=\frac{\kappa}{2 \pi}$ where $\kappa$ is the surface gravity of the black hole [2]. This thermal emission means that any hole with mass $M$ will "evaporate" in a time $\approx M^{3}$.

Thus if $M^{3}<\tau$ the Hubble time $\approx 10^{60}$ n.u. it will have vanished or at least diminished to the Planck mass by now. It is a direct consequence of the well known Cosmological Coincidences [12] that the mass at which this occurs is equal (in order of magnitude) to the value we have found above i.e. $10^{15} \mathrm{~g}$.

Thus we now see that if $M<\frac{1}{m}$ it will thermally emit particles of mass $m$ at a reasonable rate since the temperature of the Hole $T \approx \frac{1}{M}>m$. If the hole is charged then the emission factor is as if this we an additional contribution to the chemical potential of $\pm \frac{e Q}{r_{+}}$. This means that particles of the same charge to that of the hole be emitted more readily than those of opposite charge and thus leading to a net thermionic current and a neutralization of the hole. 
This process may be viewed as being due to a vacuum polarization effect and it leads naturally to the question of how black hole responds to this pair production. In ordinary electrodynamics not only are pairs formed but the external electric field is altered $[13,14]$ thus producing modifications of Coulomb's law.

This paper is mainly concerned with laying down a foundation for later studies of such processes and the calculation of the emission process. In particular we shall demonstrate that for large black holes we recover the Schwinger formula, and we calculate superradiant scattering coefficients. We shall do this by first considering the general theory of charged particles in an external gravitational electromagnetic field. For simplicity we consider spin zero particles although the extension to fermions is fairly straightforward. Then we proceed to consider the particular geometry of the black hole situation. In the next section we calculate some of the relevant scattering coefficients and in the final section make some remarks concerning their application.

\section{Theory of Vacuum Polarization in External Electromagnetic and Gravitational Fields for Spin Zero Particles}

In this section we consider a given space time with a given ( $c$ number) electromagnetic field. The theory is specified by giving field equations, commutation relations and a definition of the vacuum state. In what follows we shall assume that the spacetime possess a partial cauchy surface whose cauchy development is the region of spacetime we are interested in. We shall be working throughout the Heisenberg picture. Since we are dealing with quantum mechanical fields we require a vector potential $A_{\alpha}$ and we shall assume we are in a gauge in which

$$
A^{\alpha}{ }_{; \alpha}=0 \text {. }
$$

The particles are described by a quantum field $\phi$ which obeys the Klein-Gordan equation

$$
\left(\nabla_{\mu}-i e A_{\mu}\right)\left(\nabla^{\mu}-i e A^{\mu}\right) \boldsymbol{\phi}+m^{2} \boldsymbol{\phi}=0 .
$$

$\nabla_{\mu}$ denotes covariant differentiation. It will be convenient to introduce the gauge invariant operator $\mathscr{D}_{\mu}$ defined by

$$
\mathscr{D}_{\mu}=\nabla_{\mu}-i e \mathrm{~A}_{\mu}
$$

(2.2) implies the existence of a conserved current

$$
\boldsymbol{J}_{\mu}=\frac{e}{2 i}\left\{\boldsymbol{\phi}^{+} \mathscr{D}_{\mu} \boldsymbol{\phi}-\boldsymbol{\phi}\left(\mathscr{D}_{\mu} \boldsymbol{\phi}\right)^{+}\right\} .
$$

+ denotes hermitian conjugate, - will denote complex conjugate.

To write down the commutation relations we set

$$
\left[\phi(x), \phi\left(x^{\prime}\right)\right]=\left[\phi^{+}(x), \phi^{+}\left(x^{\prime}\right)\right]=0
$$

and note that $\left[\phi(x), \phi^{+}\left(x^{\prime}\right)\right]$ is a solution of (2.2) with respect to the variable $x$ and also the complex conjugate of (2.2) regarded as a function of $x^{\prime}$. Thus we may set

$$
\left[\phi(x), \phi^{+}\left(x^{\prime}\right)\right]=i I G\left(x, x^{\prime}\right)
$$


with the symmetry

$$
\overline{G\left(x, x^{\prime}\right)}=-G\left(x^{\prime}, x\right)
$$

and note that $G\left(x, x^{\prime}\right)$ is completely specified by giving $G\left(x, x^{\prime}\right)$ and $t^{\alpha} \mathscr{D}_{\alpha} G\left(x, x^{\prime}\right)$ on an initial surface $\Sigma$ with normal $t^{\alpha}$ which passes through $x^{\prime}$. We set

$$
\begin{aligned}
G\left(x, x^{\prime}\right) & =0, \\
t^{\alpha} \mathscr{D}_{\alpha} G\left(x, x^{\prime}\right) & =\delta\left(x, x^{\prime}\right),
\end{aligned}
$$

where $\delta\left(x, x^{\prime}\right)$ is the delta function on $\Sigma$. These properties completely determine $G\left(x, x^{\prime}\right)$ and in fact ensure that properties (2.7), (2.8) hold on any other surface through $x^{\prime} . G\left(x, x^{\prime}\right)$ is of course a "c number".

We note that both the commutation relations and the field equations and Eq. (2.1) are invariant under both charge conjugation

$$
\phi \rightarrow \boldsymbol{\phi}^{+}, \quad e \rightarrow-e
$$

and gauge transformations

$$
\phi \rightarrow e^{i e \Lambda} \phi, \quad A_{\alpha} \rightarrow A_{\alpha}+\nabla_{\alpha} \Lambda
$$

provided

$$
\nabla_{\alpha} \nabla^{\alpha} \Lambda=0 \text {. }
$$

To procede further we need to define the vacuum state. We begin by defining a scalar product between any two $c$ number solutions of (2.2) by:

$$
(\chi, \psi)=\frac{1}{2 i} \int\left(\bar{\chi} \mathscr{D}_{\mu} \psi-\psi \overline{\mathscr{D}_{\mu} \chi}\right) d \Sigma^{\mu}
$$

which enjoys the properties

$$
\begin{aligned}
\overline{(\chi, \psi)} & =(\psi, \chi), \\
(\alpha \chi, \beta \psi) & =\bar{\alpha} \beta(\chi, \psi) .
\end{aligned}
$$

$(\chi, \chi)$ is not positive definite nor does it vanish only if $x$ vanishes, its sign however cannot be altered by simply rescaling. We shall refer to $(\chi, \chi)$ as the "norm" of $\chi$ though it does not have the usual properties of a norm as used by mathematicians. Using this scalar product it is possible to introduce a complete set of $c$ number solutions of (2.2) $-\left\{p_{i}, n_{i}\right\}$ with the properties

$$
\left(p_{i}, p_{j}\right)=\delta_{i j} ; \quad\left(n_{i}, n_{j}\right)=-\delta_{i j} ; \quad\left(p_{i}, n_{j}\right)=0 .
$$

Such a set will be referred to as a basis. We have written (2.15) as if $\left\{p_{i}, n_{i}\right\}$ were a discrete set but in practice of course there will be a continuous range of such functions.

One way of constructing a basis is as follows: chose a partial cauchy surface $\Sigma$ with normal $t^{\alpha}$ and upon it chose a complete set of functions $\sigma_{i}$ normalized such that

$$
\int \sigma_{i} \bar{\sigma}_{j} d \Sigma=\delta_{i j}
$$


To specify a $p_{i}$ we need only to give $p_{i}$ and $\dot{p}_{i}=t^{\alpha} \mathscr{D}_{\alpha} p_{i}$ on $\Sigma$. This determines $p_{i}$ by the Cauchy-Kowaleski theorem - at least in a neighbourhood of $\Sigma$. Similarly for the $n_{i}$. The choice to be made is

$$
\begin{array}{ll}
p_{i}=\frac{1}{\sqrt{2 \alpha_{i}}} \sigma_{i} & \dot{p}_{i}=i \sqrt{\frac{\alpha_{i}}{2}} \sigma_{i} \\
n_{i}=\frac{1}{\sqrt{2 \beta_{i}}} \sigma_{i} & \dot{n}_{i}=-i \sqrt{\frac{\beta_{i}}{2}} \sigma_{i},
\end{array}
$$

where $\alpha_{i}, \beta_{i}$ are positive numbers chosen for convenience (often in practice to make simple separable solutions). To see that these are complete we note that any solution $\psi$ of (2.2) is specified by $\psi$ and $\dot{\psi}$ on $\Sigma$.

Let

$$
\psi=\sum_{i} e_{i} \sigma_{i} ; \quad \dot{\psi}=\sum_{i} f_{i} \sigma_{i}
$$

if

$$
\begin{aligned}
& \psi=\sum_{i} a_{i} p_{i}+c_{i} n_{i} \quad \text { then on } \Sigma \\
& \psi=\sum_{i}\left(\frac{a_{i}}{\sqrt{2 \alpha i}}+\frac{c_{i}}{\sqrt{2 \beta_{i}}}\right) \sigma_{i} \quad \dot{\psi}=\sum_{i} i\left(a_{i} \sqrt{\frac{\alpha_{i}}{2}}-c_{i} \sqrt{\frac{\beta_{i}}{2}}\right) \sigma_{i} .
\end{aligned}
$$

These equations are sufficient to determine the $a$ 's and $c$ 's in terms of the $e$ 's and $f$ 's.

Having constructed a basis we may expand the quantum field operator as

$$
\phi=\sum_{i}\left(\boldsymbol{a}_{i} p_{i}+\boldsymbol{c}_{i} n_{i}\right)
$$

Where the $\boldsymbol{a}$ 's and $\boldsymbol{c}$ 's are position independent operators. In order to satisfy the commutation relations it is sufficient to set

$$
\left[\boldsymbol{a}_{i}, \boldsymbol{a}_{k}^{+}\right]=\delta_{i k} \quad\left[c_{i}, \boldsymbol{c}_{k}^{+}\right]=-\delta_{i k}
$$

or with $\boldsymbol{c}_{i}=\boldsymbol{b}_{i}^{+}$

$$
\left[\boldsymbol{b}_{i}, \boldsymbol{b}_{k}^{+}\right]=\delta_{i k}
$$

and zero for all other commutators.

The $\boldsymbol{a}$ 's and $\boldsymbol{b}$ 's are of course annihilation and creation operators for "particle" and "antiparticle" states described by the $p$ 's and $n$ 's respectively. However that statement has no invariant meaning because one is at liberty to chose a new basis $\left\{p_{i}^{\prime}, n_{i}^{\prime}\right\}$ related to the old one by:

$$
\begin{aligned}
& p_{i}^{\prime}=\sum_{j}\left\{\alpha_{i j}^{(+)} p_{j}+\beta_{i j}^{(+)} n_{j}\right\}, \\
& n_{i}^{\prime}=\sum_{j}\left\{\alpha_{i j}^{(-)} n_{j}+\beta_{i j}^{(-)} p_{j}\right\},
\end{aligned}
$$

with

$$
\begin{aligned}
\delta_{i R} & =\alpha_{i j}^{(+)} \overline{\alpha_{k j}^{(+)}}-\beta_{i j}^{(+)} \overline{\beta_{k j}^{(+)}}, \\
\delta_{i R} & =\alpha_{i j}^{(-)} \overline{\alpha_{k j}^{(-)}}-\beta_{i j}^{(-)} \overline{\beta_{k j}^{(-)}}, \\
0 & =\alpha_{i j}^{(+)} \overline{\beta_{k j}^{(-)}}-\beta_{i j}^{(+)} \overline{\alpha_{k j}^{(-)}} .
\end{aligned}
$$


This induces a Bogoliubov transformation on the $a_{i}$ and $b_{i}$ leaving the commutation relations unchanged as follows

$$
\begin{aligned}
& \boldsymbol{a}_{i}^{\prime}=\sum_{j} \overline{\alpha_{i j}^{(+)}} \boldsymbol{a}_{j}-\overline{\beta_{i j}^{(+)}} \boldsymbol{b}_{j}^{\dagger}, \\
& \boldsymbol{b}_{i}^{\prime}=\sum_{j} \overline{\alpha_{i j}^{(-)}} \boldsymbol{b}_{j}-\overline{\beta_{i j}^{(-)}} \boldsymbol{a}_{j}^{\dagger} .
\end{aligned}
$$

Had we defined a vacuum state $|0\rangle$ by the conditions

$$
\begin{aligned}
& \boldsymbol{a}_{i}|0\rangle=0, \\
& \boldsymbol{b}_{i}|0\rangle=0 .
\end{aligned}
$$

These equations would not remain true in the new basis since provided $\beta_{i j}^{(+)}$and $\beta_{i j}^{(-)} \neq 0$ we shall have mixed in some creation operators into the new annihilation operators.

If there exists a symmetry of the space time which is also a symmetry of the electromagnetic field, generated by a killing vector $K_{\alpha}$ say one may construct a global, generalized momentum operator $\boldsymbol{K}$ which is well defined by the following prescription. Let $\boldsymbol{T}_{\alpha \beta}$ be the symmetrized energy momentum operator-obtained by functionally differentiating the lagrangian with respect to the metric then the object

$$
K=\int T_{\alpha \beta} K^{\alpha} d \Sigma^{\beta},
$$

where the integration extends over any partial cauchy surface is independent of that surface provided $\phi$ falls off sufficiently rapidly at infinity since

$$
\left(T_{\alpha \beta} K^{\beta}\right)^{; \alpha}=0 .
$$

It generates transformations on the operator $\phi$ in the sense that it is an infinitesimal generator of unitary transformations on the space of operators and obeys:

$$
i[\boldsymbol{\phi}, \boldsymbol{K}]=\underset{\boldsymbol{K}_{\alpha}}{\mathscr{L}} \Phi
$$

where $\mathscr{L}$ is the Lie derivative.

One is also at liberty to chose solutions of (2.2) which have an exponential dependence on the corresponding group parameter i.e. such that

$$
\underset{K_{\alpha}}{\mathscr{L}} \phi=i \alpha \phi,
$$

where $\alpha$ is a constant. These solutions will act as raising and lowering operators in the sense that if $|K\rangle$ is an eigenstate of $\boldsymbol{K}$ with eigenvalue $K$ then $\boldsymbol{\phi}^{\dagger}|K\rangle$ is a field of eigenstates of $K$ with eigenvalue $K+\alpha$ and $\phi|K\rangle$ a field of eigenstates with eigenvalue $K-\alpha$. If $L_{\alpha}$ is another commuting killing vector we may consider solutions which also obey:

$$
\underset{L_{\alpha}}{\mathscr{L}} \boldsymbol{\phi}=i \beta \boldsymbol{\phi} .
$$

If solutions of this type are chosen to make a basis then the creation operation will create particle states with generalized momentum $\alpha$ and $\beta$. If the spacetime and the electromagnetic field are invariant under an $r$ parameter group $G_{r}$ which is not necessarily abelian we can label the killing vectors with upper case latin letters thus $K_{A}^{\alpha}$. A ranges from 1 to $r$. 
The structure constants of the group $C_{A B}^{C}$ defined by

$$
\left(\begin{array}{l}
\mathscr{L} \underset{K_{A}}{\mathscr{L}} \underset{K_{B}}{\mathscr{L}}-\underset{K_{B}}{\mathscr{L}} \underset{K_{A}}{\mathscr{L}}
\end{array}\right) \phi=C_{A{ }_{K_{C}}^{C}}^{\mathscr{L}} \phi
$$

imply the commutation relations

$$
\left[\boldsymbol{K}_{A}, \boldsymbol{K}_{B}\right]=i C_{A B}^{C} \boldsymbol{K}_{C}
$$

for the corresponding generalized momentum operators. Thus if $r$ of the killing vectors commute $(r \leqq 4)$ we may take solutions with exponential dependence on the $r$ group parameters (if $r=4$ we have the field free, flat space case). We could also consider operators which we built up quadratically from killing vectors. For example if there is spherical symmetry we can consider the "total angular momentum operator" $L^{2}$ defined by

$$
\boldsymbol{L}^{2}=\boldsymbol{L}_{x}^{2}+\boldsymbol{L}_{y}^{2}+\boldsymbol{L}_{2}^{2},
$$

where $L_{x}$ etc. are the operators built out of the killing vectors generating rotations about three orthogonal axes. Corresponding to Eq. (2.34) we can write

$$
-\left[\phi, L^{2}\right]=\underset{L_{x}^{\alpha}}{\mathscr{L}} \underset{L_{x}^{\alpha}}{\mathscr{L}} \phi+\underset{L_{y}^{\alpha}}{\mathscr{L}} \underset{L_{y}^{\alpha}}{\mathscr{L}} \phi+\underset{L_{z}^{\alpha}}{\mathscr{L}} \underset{L_{z}^{\alpha}}{\mathscr{L}} \phi
$$

In the same way as before we can consider solutions of (2.2) which obey for example

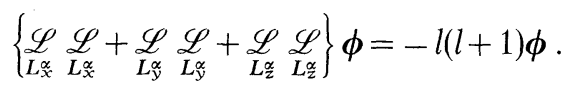

And in a similar way as before these solutions will act as lowering and raising operators for total angular momentum. The reason for doing this in flat space field free is of course to construct a vacuum state which is invariant under the group of motions of flat space - that is the Poincare group. This is done by fourier analyzing, i.e. using the 4 commuting translation operators. However there is not quite sufficient - one must also modify the definition (2.29) by subtracting off the vacuum expectation value of the momentum operators (normal ordering). This will of course not affect Eq. (2.31) An alternative procedure in flat field free space is to use the time translations and generators of angular momentum about one axis and total angular momentum. This choice of basis is equivalent to the usual one since there is no mixing of positive and negative frequencies $\left(\beta_{i j}^{(+)}=\beta_{i j}^{(-)}=0\right.$ ) and Eqs. (2.25) and (2.26) reduce to a unitary transformation of the annihilation and creation operators. If on the other hand one uses a different timelike killing vector - for example the generator of boosts in certain direction one will obtain a basis which is not merely a unitary transformation of the usual one (i.e. $\beta_{i j}^{(+)}=0$ ) and one's definition of the particle states is altered (cf. [15]).

In spacetimes which become in some sense asymptotically flat and field free it is possible to use a basis which tends in the asymptotic region to the usual basis in flat, field free space. This is the usual approach of $S$-matrix theory. If on the other hand there is a lot of symmetry present one may be inclined to make use of it in defining the vacuum. If there is no asymptotically flat region one may have to. In any case the choice of the vacuum is something that has to be decided on physical grounds and is not provided by the formalism. However once we have chosen a vacuum we may procede to calculate for example the expectation value 
of the current. It is easy to see that this reduces to

$$
\left\langle 0\left|\boldsymbol{J}_{\mu}\right| 0\right\rangle=e \sum_{i} \bar{n}_{i}\left(\mathscr{D}_{\mu} n_{i}\right)-p_{i} \overline{\left(\mathscr{D}_{\mu} p_{i}\right)} \text {. }
$$

If $A_{\mu}=0$ we have $\mathscr{D}_{\mu}=\overline{\mathscr{D}}_{\mu}$ and $\bar{n}_{i}$ can be taken as $p_{i}$ so that the current vanishes. The application of an external field effects the "virtual" particles and antiparticles in the vacuum differently, causing currents to flow and charge distributions to build up which will in turn modify the external field even though Eq.(2.15) guarantee overall neutrality. It is of interest to calculate the vacuum current in terms of another basis. A short calculation leads to:

$$
\begin{aligned}
\left\langle 0\left|\boldsymbol{J}_{\alpha}\right| 0\right\rangle= & e \sum_{i}\left\{\bar{n}_{i}^{\prime} \mathscr{D}_{\alpha} n_{i}^{\prime}-p_{i}^{\prime} \overline{\left(\mathscr{D} \alpha p_{i}^{\prime}\right)}\right\} \\
& +\sum_{i, j, k} e \beta_{j i}^{(-)} \overline{\beta_{k i}^{(-)}}\left\{\bar{n}_{i}^{\prime} \mathscr{D}_{\alpha} n_{k}^{\prime}-n_{j} \overline{\mathscr{D}_{\alpha} n_{k}^{\prime}}\right\} \\
& \sum_{i, j, k} e \beta_{j i}^{(+)} \overline{\beta_{k i}^{(+)}}\left\{p_{j}^{\prime} \overline{\mathscr{D}_{\alpha} p_{k}^{\prime}}-\bar{p}_{j}^{\prime} \mathscr{D}_{\alpha} p_{k}^{\prime}\right\} \\
& +\sum_{i, j, k} e \overline{\beta_{j i}^{(-)}} \alpha_{k i}^{(+)}\left\{n_{j}^{\prime} \overline{\mathscr{D}_{\alpha} p_{k}^{\prime}}-\bar{p}_{j}^{\prime} \overline{\mathscr{D}_{\alpha} n_{k}^{\prime}}\right\} \\
& +\sum_{i, j, k} e \overline{\beta_{j i}^{(+)}} \alpha_{k i}^{(-)}\left\{p_{j}^{\prime} \overline{\mathscr{D}_{\alpha} n_{k}^{\prime}}-\bar{n}_{j}^{\prime} \mathscr{D}_{\alpha} p_{k}^{\prime}\right\} .
\end{aligned}
$$

\section{Geometry of Collapse and Choice of Vacuum State}

In order to determine whether the black hole loses charge and how fast it is necessary to integrate the vacuum current over a surface at infinity. We could of course use expression (2.34) directly but it is more convenient to introduce a new basis at large future times which can be separated into a set of positive and negative norm functions which are zero on the future horizon and purely outgoing at infinity which we write as $\left\{p_{i}\left(\mathscr{I}^{+}, I^{+}\right), n_{i}\left(\mathscr{I}^{+}, I^{+}\right)\right\}$and a set which carry no flux to infinity and are purely ingoing on the future horizon which we write as $\left\{p_{i}\left(\mathscr{H}^{+}\right), n_{i}\left(\mathscr{H}^{+}\right)\right\}$. To completely specify them we chose them to have exponential time dependence and to be multiples of spherical harmonics. Since near infinity we expect the solution to look approximately like the exact Reisner-Nordstrom solution this is permissible. If we then calculate the total charge lost to infinity infinity using expression (2.35) we find it to be

$$
\sum_{i, j} e\left\{\left|\beta_{i j}^{(+)}\right|^{2}-\left|\beta_{i j}^{(-)}\right|^{2}\right\} .
$$

The $i$ summation running only over the set $\left\{p_{i}\left(\mathscr{I}^{+}, I^{+}\right), n_{i}\left(\mathscr{I}^{+} . I^{+}\right)\right\}$. The important question we have to answer now is what basis do we use to describe the initial state? This depends upon amongst other things how one envisages the collapse. One approach is to use the exact geometry of the Reisner-Nordstrom solution whose Penrose diagram is shown in Fig. 2. Here we are only concerned with the shaded exterior region. For this region $\Sigma$ may be taken as a cauchy surface. The basis is chosen to consist of functions which are outgoing on the past horizon and carry no flux through past infinity (i.e. $\left.I^{-} \cup \mathscr{I}^{-}\right)-\left\{p_{i}\left(\mathscr{H}^{-}\right), n_{i}\left(\mathscr{H}^{-}\right)\right\}$and a set which on zero on $\mathscr{H}^{-}$and ingoing at past infinity $-\left\{p_{i}\left(\mathscr{I}^{-}, I^{-}\right), n_{i}\left(\mathscr{I}^{-}, I^{-}\right)\right\}$. Since there is both time and spherical symmetry the solutions are also chosen to be products of spherical harmonics and time dependence exp iwt. We find that

$$
\begin{array}{llll}
p_{i}\left(\mathscr{I}^{+}, I^{+}\right), & p_{i}\left(\mathscr{I}^{-}, I^{-}\right) & \text {have } & \omega>0 \\
n_{i}\left(\mathscr{I}^{+}, I^{+}\right), & n_{i}\left(\mathscr{I}^{-}, I^{-}\right) & \text {have } & \omega<0 .
\end{array}
$$




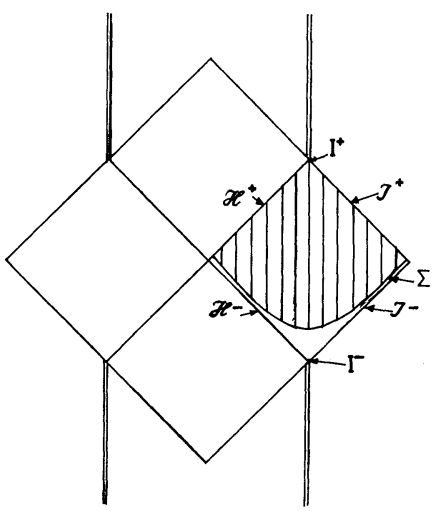

Fig. 2. The Penrose diagram for the Reisner-Nordstrom solution. Each point represents a 2-sphere. Null lines are at $45^{\circ}$. The dotted line $\Sigma$ represents a possible cauchy surface for the shaded exterior region $\mathscr{H}^{+}$and $\mathscr{H}^{-}$are the future and past event horizons, $\mathscr{I}^{+}$and $\mathscr{I}^{-}$future and past null infinity and $I^{+}, I^{-}$future and past timelike infinity

But

$$
\begin{aligned}
& p_{i}\left(\mathscr{H}^{-}\right) \text {have } \omega+\frac{e Q}{r_{+}}>0 \\
& n_{i}\left(\mathscr{H}^{-}\right) \text {have } \omega+\frac{e Q}{r_{+}}<0
\end{aligned}
$$

as will be seen in the next section. Those modes with negative frequencies smaller in magnitude than $\frac{e Q}{r_{+}}$can be members of both the sets $p_{i}\left(\mathscr{H}^{-}\right)$and $n_{i}\left(\mathscr{I}^{+}, I^{+}\right)$ and will contribute to the sum in Eq. (3.1). In order to propagate to infinity we must have

$$
\omega^{2}>m^{2} \text {. }
$$

Thus the modes in question are just the superradiant modes whose existence depends on criterion (1.2). It is now possible on this picture to resolve the paradox of how a static field can produce particles to infinity. Normally one says that particle production is due to the mixing of positive and negative frequencies by a time varying field, since particle states are defined by positive frequencies and antiparticle states vice versa. If instead one includes the electromagnetic field into ones definition of particle and antiparticle this will not be true - criterion like (3.2) and (3.3) will hold and the paradox is resolved. This is the same phenomenon as was discovered many years ago by Snyder and Weinberg [16] in the case of a deep, time independent well in flat space. There the situation was ascribed to the failure of the Hamiltonian operator and the total charge operator,

$$
\boldsymbol{Q}=\int \boldsymbol{J}^{\mu} d \Sigma_{\mu}
$$

to commute. This is just what is happening here.

The sum in Eq. (3.1) will, in practice be infinite but this will, as in $[8,9]$, correspond to a steady rate of emission of particles. In this case the quantity $-\sum_{i}\left|\beta_{i j}^{(-)}\right|^{2}$ measures how much flux a unit wave at future infinity carries into the past event 


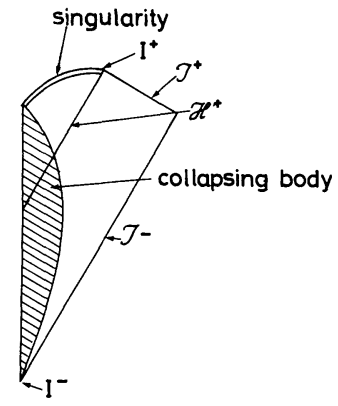

Fig. 3. The Penrose diagram for the gravitational collapse of a charged fluid

horizon - i.e. it is the absorption coeff., $A_{i}^{(-)}$. If we convert all the quantities to rates by averaging the flux over a large time we obtain for the rate of loss of charge the sum of the superradiant coefficients that is:

$$
\frac{d Q}{d t}=e \sum_{i} A_{i}^{(-)} .
$$

The summation being only over the superradiant modes. Since for superradiant modes $A_{i}^{(-)}$is negative the hole loses charge.

The drawback with this method is that in a realistic gravitational collapse the past event horizon will not exist. It is this which motivates Hawking's choice of the initial no particle state. This is best seen from Fig. 3 which is the Penrose diagram of an idealized spherically symmetric collapse of a charged fluid. As a cauchy surface he choses $I^{-} \cup \mathscr{I}^{-}$and as a basis the set of ingoing waves with time dependence expict. The time dependence of the gravitational collapse mixes the positive and negative frequencies and he obtains an expression similar to (3.1), for the total charge emitted. The remarkable fact about his analysis is that he is able to show that the expressions $\sum_{j}\left|\beta_{i j}^{(+)}\right|^{2}$ and $\sum_{j}\left|\beta_{i j}^{(-)}\right|^{2}$ when converted to a rate as above has the form:

$$
\frac{A_{i}^{(+)}}{\exp \left(\omega_{i}+\frac{e Q}{r_{+}}\right) / T-1} \text { and } \frac{A_{i}^{(-)}}{\exp \left(\omega_{i}-\frac{e Q}{r_{+}}\right) / T-1}
$$

where $A_{i}^{(+)}$and $A_{i}^{(-)}$are the absorption coefficients for positive and negative frequencies (that is positive and negative charges) in the exact Reisner-Nordstrom geometry at a frequency $\omega_{i}$ and

$$
T=\frac{\kappa}{2 \pi} .
$$

The result that one obtains for the rate of loss of charge is then

$$
\frac{d Q}{d t}=e \sum_{i} \frac{A_{i}^{(+)}}{\exp \left(\omega_{i}+\frac{e Q}{r_{+}}\right) / T-1}-\frac{A_{i}^{(-)}}{\exp \left(\omega_{i}-\frac{e Q}{r_{+}}\right) / T-1}
$$


note that $A_{i}^{(-)}$is positive for $\omega_{i}>\frac{e Q}{r_{+}}$and negative for the superradiant modes, $\omega_{i}<\frac{e Q}{r_{+}}$. As Hawking has pointed out in the limit of very low temperatures, for example for very massive black holes the collapse is very slow and expression (3.6) goes over into (3.5). It also agrees with the result one would get by applying Schwinger's formula as will be seen later. The advantage of deriving the results in the way we have is that expression (2.29) for the vacuum current will be valid everywhere in the region outside the hole and on the future horizon whereas looking at the rate of particles emitted at future infinity gives no local information about the vacuum current.

Contrasting the two methods, Unruh's and Hawking's we see that Hawking's corresponds to switching the interactions off at past infinity and Unruh's to keeping it on. As such both methods could be applied any problem in field theory. On the whole it seems to the author that the latter (switching the interaction off at past infinity) is more appropriate in most, "asymptotically flat problems" including the production of pairs by a potential well. In a cosmological context where there exists no asymptotically flat region the answer is less obvious.

\section{Calculation of Superradiant Coefficients}

From what has been said in the previous section it is clear that the calculation of the absorbtion and superradiant coefficients is essential to understand the loss of charge. In this section we provide some approximate forms for these coefficients and derive some of the results quoted above. We begin by noting that the KleinGordan equation is separable in the usual coordinates for the Reisner-Nordstrom solution and we may consider solutions of the form

$$
\Phi=e^{i \omega t} Y_{i m}(\theta, \phi) R(r)
$$

then $R$ will obey the equation

$$
\Delta r^{2} \frac{d}{d r}\left(r^{2} \Delta \frac{d R}{d r}\right)+\left\{\left(\omega+\frac{e Q}{r}\right)^{2}-\left(m^{2}+\frac{l(l+1)}{r^{2}}\right) \Delta\right\} r^{4} R=0
$$

we are taking

$$
\begin{aligned}
\Delta & =g_{00}=1-\frac{2 M}{r}+\frac{Q^{2}}{r^{2}}, \\
A_{\alpha} d x^{\alpha} & =\frac{Q}{r} d t .
\end{aligned}
$$

Equation (4.2) has 2 regular singular points at $r=r_{+} ; r=r_{-}$, the 2 two roots of $\Delta$ and an irregular singular point at infinity, surprisingly perhaps $r=0$ is a regular point. (4.2) does not have solutions in terms of known or tabulated functions apart from the exceptional case's $\omega^{2}=m^{2}=0$ and $\omega^{2}=m^{2}=e^{2} Q^{2} M^{-2}$ when the singularity at $r=\infty$ becomes regular and the equation may be solved in terms of 
hypergeometric functions. Since this case is a little interest we shall not do this but turn to the boundary conditions to be satisfied by solutions of (4.2). At infinity the asymptotic form of the solutions is

$$
R=\frac{1}{r} \exp \pm i\{k r-\alpha \log 2 R r\}
$$

With $R=\sqrt{\omega^{2}-n^{2}}$ and $\alpha=-\left(M\left(2 \omega^{2}-m^{2}\right)+e \omega Q\right) k^{-1}$. If $k$ is real and we agree to take the positive square root then for positive frequencies the positive sign indicates ingoing waves and the negative sign outgoing waves. If the frequency is negative then the contrary is true. If $k$ is complex then one solution grows exponentially and one decays exponential with $r$. Near $r=r_{+}$the solutions are:

$$
\frac{1}{r} \exp \pm i\left(\omega+\frac{e Q}{r_{+}}\right) y \quad \text { times analytic function of }\left(r-r_{+}\right)
$$

with

$$
y=r+\frac{r_{+}^{2}}{\left(r_{+}-r_{-}\right)} \log \left(r-r_{+}\right)-\frac{r_{-}^{2}}{\left(r_{+}-r_{-}\right)} \log \left(r-r_{-}\right) .
$$

As $r$ ranges between $r_{+}$and $\infty, y$ ranges between $-\infty$ and $+\infty$. To discuss the boundary conditions at the horizons it is necessary to introduce a set of coordinates and a new vector potential related to the old one by a gauge transformation which are well behaved on the horizons. For the future horizon the new coordinate required is the advanced time coordinate $v$ defined by

$$
v=t+y .
$$

The new vector potential $\tilde{A}_{\mu}$ is generated by the function

$$
\tilde{\Lambda}=-\frac{e Q}{r_{+}} \frac{1}{2 \kappa}\left\{\log \left(r-r_{+}\right)-\log \left(r-r_{-}\right)\right\} .
$$

The transformed function $\tilde{\Phi}$ is given by:

$$
\tilde{\Phi}=Y_{e m} R \exp \left\{i \omega v+i \omega r+\frac{i}{2 k}\left(\frac{e Q}{r_{+}}+\omega\left(\frac{r_{-}}{r_{+}}\right)^{2}\right) \log \left(r-r_{+}\right)-\frac{i}{2 k}\left(\omega+\frac{e Q}{r_{+}}\right) \log \left(r-r_{+}\right)\right\} .
$$

Thus near $\mathscr{H}^{+}$

$$
\tilde{\Phi} \propto \exp \left\{\frac{-i}{2 \kappa}\left(\omega+\frac{e Q}{r_{+}}\right)( \pm 1-1) \log \left(r-r_{+}\right)\right\} \times \text {analytic function of }\left(r-r_{+}\right) .
$$

$\tilde{\Phi}$ will be analytic if we take the positive sign (ingoing waves). If we take the positive sign (outgoing waves) the solution will be $C^{p}$ iff

$$
\mathscr{I}_{m}\left(\frac{\omega}{\kappa}\right)>p \text {. }
$$


On the past horizon we introduce a retarded time coordinate $u$ defined by

$$
u=t-y
$$

and a new vector potential $\tilde{A}_{\mu}$ generated by the function

$$
\tilde{\Lambda}=-\tilde{\Lambda} \text {. }
$$

The new function $\tilde{\tilde{\Phi}}$ will be analytic near $\mathscr{H}^{-}$if we take the negative sign (outgoing) and $C^{p}$ iff $\mathscr{I}_{m} \frac{\omega}{\kappa}<p$.

If $l^{\alpha}\left(n^{\alpha}\right)$ are the future directed normals of the future (past) horizons then the gauge invariant derivatives obey, up to an overall positive factor which depends on the scaling of $l^{\alpha}$ and $n^{\alpha}$

$$
\begin{gathered}
l^{\alpha} \tilde{\mathscr{D}_{\alpha}} \tilde{\Phi}=i\left(\omega+\frac{e Q}{r_{+}}\right) \tilde{\Phi}=i v \tilde{\Phi} \\
n^{\alpha} \tilde{\tilde{D}}{ }_{\alpha} \tilde{\Phi}=i\left(\omega+\frac{e Q}{r_{+}}\right) \tilde{\Phi}=i v \tilde{\Phi} .
\end{gathered}
$$

On the horizon the dependence of $\tilde{\Phi}, \tilde{\Phi}$ on the coordinates in the horizons is

$$
e^{i v v} Y_{l m} \text { and } e^{i v v} Y_{l m} \text {. }
$$

Confining ourselves to real frequencies obeying $\omega^{2}>m^{2}$ we see that set $\left\{p_{i}\left(\mathscr{I}^{-}, I^{-}\right)\right\}$ corresponds to $\omega>m$ and $R$ having asymptotic form:

$$
\begin{aligned}
& R \rightarrow \frac{1}{k r} \exp (i k r)+f^{+} \frac{1}{k r} \exp (-i k r) \text { as } r \rightarrow \infty \\
& R \rightarrow \frac{g^{+}}{r_{+}} \exp +-i\left(\omega+\frac{e Q}{r_{+}}\right) y \text { as } r \rightarrow r_{+} .
\end{aligned}
$$

Using the Wronskian of (4.2) one can show that

$$
1-\left|f^{+}\right|^{2}=\left(\omega+\frac{e Q}{r_{+}}\right)\left|g^{+}\right|^{2}=A_{k}^{(+)} \text {. }
$$

The set $\left\{n_{i}\left(\mathscr{I}^{-}, I^{-}\right)\right\}$have $\omega<-m$ and $R$ with asymptotic form:

$$
\begin{aligned}
& R \rightarrow \frac{1}{R r} \exp (-i k r)+f^{-} \frac{1}{R r} \exp (+i k r) \text { as } r \rightarrow \infty \\
& R \rightarrow \frac{g^{-}}{r_{+}} \exp +i\left(\omega+\frac{e Q}{2}\right) y
\end{aligned}
$$

and

$$
1-\left|f^{-}\right|^{2}=-\left(\omega+\frac{e Q}{r_{+}}\right)\left|g^{-}\right|^{2} .
$$

The normalization is such that

$$
\left(p_{k}\left(\mathscr{I}^{-}, I^{-}\right), p_{k}\left(\mathscr{I}^{-}, I^{-}\right)\right)=\delta\left(k-k^{\prime}\right) \delta_{m m^{\prime}} \delta_{u u^{\prime}} \quad \text { e.t.c . }
$$


The set $\left\{p_{i}\left(\mathscr{I}^{+}, I^{+}\right)\right\}$have: $\omega>m$ and with asymptotic form:

$$
\begin{aligned}
& R \rightarrow \frac{1}{k r} \exp -i k r+h^{+} \frac{1}{k r} \exp (+i k r) \text { as } r \rightarrow \infty \\
& R \rightarrow j^{+} \frac{1}{r_{+}} \exp -i\left(\omega+\frac{e Q}{r_{+}}\right) y \text { as } r \rightarrow r_{+}
\end{aligned}
$$

and $\left\{n_{i}\left(\mathscr{I}^{+}, I^{+}\right)\right\}$have: $\omega<-m$

$$
\begin{aligned}
& R \rightarrow \frac{1}{k r} \exp (+i k r)+h^{-} \frac{1}{k r} \exp (-i k r) \text { as } r \rightarrow \infty \\
& R \rightarrow \frac{j^{-}}{r_{+}} \exp -i\left(\omega+\frac{e Q}{r_{+}}\right) y \text { as } r \rightarrow \infty .
\end{aligned}
$$

The set $\left\{p_{i}\left(\mathscr{H}^{-}\right)\right\}$have $\omega+\frac{e Q}{r_{+}}>0$ and

$$
R \rightarrow \frac{1}{r_{+}} \frac{1}{\sqrt{v}} \exp i v y+\frac{s^{+}}{r_{+}} \frac{1}{\sqrt{v}} \exp -i v y \quad \text { as } r \rightarrow r_{+}
$$

and

$$
\begin{aligned}
& R \rightarrow \frac{t^{+} e^{-i k r}}{k r} \text { as } \quad r \rightarrow \infty \text { if } \quad \omega>0 \\
& R \rightarrow \frac{t^{+} e^{+i k r}}{k r} \text { as } \quad r \rightarrow \infty \text { if } \quad \omega<0 .
\end{aligned}
$$

The set $\left\{n_{i}\left(\mathscr{H}^{-}\right)\right\}$have $\left(\omega+\frac{e Q}{r_{+}}\right)<0$ and

$$
R \rightarrow \frac{t^{-} e^{+i k r}}{k r} \text { as } r \rightarrow \infty
$$

Wronskian arguments yield:

$$
\begin{aligned}
& 1-\left|s^{-}\right|^{2}=\left|t^{-}\right|^{2} \\
& 1-\left|s^{+}\right|^{2}=\operatorname{sign} \omega\left|t^{+}\right|^{2} .
\end{aligned}
$$

It remains to discuss solutions of (4.2) for values of $\omega$ outside the range: real $\omega$ and $\omega^{2}>m^{2}$. That is we seek solutions which are exponentially damped at large $r$ and ingoing on the past or future horizon. If we assume that solutions exist for real $\omega$ we make use of the Wronskian of (4.2) to show that $\left(\omega+\frac{e Q}{r_{+}}\right)=0$ and so apart from the possible exceptional case $\omega=-\frac{e Q}{r_{+}}$we have a contradiction. If we assume that there are solutions which decrease exponentially with $r$, are ingoing on $\mathscr{H}^{+}$and grow exponentially with time we obtain by integrating Eq. (4.2) by parts and taking the imaginary part:

$$
\mathscr{I} m \omega \int_{r=r_{+}}^{\infty}\left(\operatorname{Re} \omega+\frac{e Q}{r}\right) \frac{|R|^{2}}{r^{2}} \frac{d r}{\Delta}=0
$$


If $e Q=0$ this is sufficient to rule out such solutions. If $e Q \neq 0$ we may rule out all such solutions with $\operatorname{Re}(\omega)>0$

$$
\text { or } \operatorname{Re}(\omega)<-\frac{e Q}{r_{+}} \text {. }
$$

We may also rule out solutions which outgoing on $\mathscr{H}^{-}$exponentially decrease with time under the same conditions.

These results may be looked at physically as follows. Equation (4.2) is of the form of a wave equation with a potential well surrounded by the usual barrier at infinity and another barrier in front of $r=r_{+}$. Thus one would expect solutions corresponding to quasi bound states which trickle through the future horizon and decay with time. These would represent particles moving in Bohr like orbits around the black hole which eventually fall into it. Since the situation is time symmetric we would also expect solutions representing waves being sent in form the past horizon and growing with time. The results above do not quite rigorously establish this but from now on we shall assume that this is so, and that modes that are ingoing at $\mathscr{H}^{+}$but grow with time are ruled out. Various work on wave equations in black hole backgrounds looking for unstable modes lends indirect support to the idea that they do not exist. The question now is whether "the quasi bound states" should be included in the basis that defines the vacuum. If we use the Hawking picture the answer is no. If the initial configuration is sufficiently dispered they will not exist if they do exist then at large future times they will be negligible in magnitude and thus will not contribute to the outward flow of particles at future infinity. On the alternative Unruh picture the situation is perhaps not so obvious, but since the decaying solutions will blow up at past infinity, they presumably will not be required in a basis for well behaved solution at past infinity.

We now proceed to calculate the scattering coefficients in two separate regimes.

\section{Large Black Holes $M m \gg 1$}

In this case we use the W.K.B. approximation. Setting $R=\frac{S}{r}$ we obtain:

$$
\frac{d^{2} S}{d \mu^{2}}+\left\{\left[\omega+\frac{e Q}{r}\right)^{2}-\left(m^{2}+\frac{l(l+1)}{r^{2}}+\frac{1}{r} \frac{d \Delta}{d r}\right) \Delta\right\} S=0 .
$$

Since $M m \gg 1$ we may replace this equation by

$$
\frac{d^{2} S}{d \mu^{2}}+W S
$$

with

$$
W=\left(\omega+\frac{e Q}{r}\right)^{2}-m^{2} \Delta .
$$

for superradiant modes $W$ has 2 roots outside $r=r_{+}, \alpha, \beta$ say. Waves which penetrate the barrier carry negative flux down the hole. The superradiant coefficient will thus be

$$
A^{(-)}=(-1) \times(\text { transmission factor })
$$


which is given by [17]

$$
\exp -2 \int_{\alpha}^{\beta} \sqrt{W} d y .
$$

The barrier integral is easily done yielding

$$
\begin{aligned}
\int_{\alpha}^{\beta} \sqrt{W} d y & =\pi e\left(1-\sqrt{1-\frac{m^{2}}{e^{2}}}\right) \frac{r_{+}^{2}}{Q} \approx \frac{\pi m^{2} r_{+}^{2}}{2 e Q}, \text { since } m^{2} \ll e^{2} \\
A^{(-)} & =\exp -\frac{\pi m^{2} r_{+}^{2}}{e Q} .
\end{aligned}
$$

This approximation will be valid for

$$
\frac{l(l+1)}{r_{+}^{2}} \ll \omega^{2} \text { and } \quad \frac{e Q}{r_{+}} \gg m .
$$

For values of greater than $\omega r_{+}$the waves experience a centrifugal barrier and the transmission factor will be much diminished. If $\frac{e Q}{r_{+}} \approx m$ the W.K.B. approximation ceases to be valid.

$$
\begin{aligned}
\frac{d Q}{d t} & =e \sum_{l, m} \int_{m}^{\frac{e Q}{r_{+}}} A^{(-)}(\omega) d \omega \\
& \approx \frac{e^{4} Q^{3}}{r_{+}} \exp -\pi \frac{\mathscr{E}_{c}}{\mathscr{E}^{\circ}}
\end{aligned}
$$

with $\mathscr{E}=\frac{e Q}{r_{+}^{2}} ;$ and $\mathscr{E}_{c}=\frac{m^{2}}{e}$.

This is the result you would expect on the basis of Schwinger's formula.

\section{Small Black Holes $M m \ll 1$}

In this case the W.K.B. method is inappropriate and we shall adopt an approximation scheme introduced into Black Hole theory by Starobinsky $[10,16]$. We begin by noting that with the substitution $x=\frac{r-r_{+}}{r_{+}-r_{-}}$Eq. (4.2) takes the form:

$$
\begin{aligned}
& x(x+1) \frac{d}{d x}\left(x(x+1) \frac{d R}{d x}\right)-\left(l(l+1)+F^{2}+W^{\prime}\right) R=0 \\
& F=\frac{1}{2 \kappa}\left(\omega+\frac{e Q}{r_{+}}\right) ; \quad \eta=\frac{r_{+}-r_{-}}{r_{+}} ; \quad B=\left(\omega^{2}-m^{2}\right) r_{+}^{2}
\end{aligned}
$$

and

$$
\begin{aligned}
A & =\frac{\eta F \omega}{\kappa}-\frac{1}{4} \eta^{2}\left(\frac{\omega}{\kappa}\right)^{2} \\
W^{\prime} & =(1+\eta x)^{2}\left\{F^{2}+A x+B x(x+1)\right\}-F^{2} .
\end{aligned}
$$


Near $x=0$ and if $F^{2} \ll 1 ; x B \ll l$ and $x A \ll 1$ we neglect $W^{\prime}$ and solve (4.29) to give

$$
R=\left(\frac{x}{x+1}\right)^{i F} F(-l ; l+1 ; 1-2 i F ; x+1) .
$$

This involves $\omega M \ll 1$. For large $x$ Eq. (4.2) may be approximated as:

$$
\frac{1}{\varrho} \frac{d}{d \varrho}\left(\varrho^{2} \frac{d R}{d \varrho}\right)-\left\{\frac{l(l+1)}{\varrho^{2}}+\frac{2 \alpha}{\varrho}-1\right\} R=0
$$

with $\varrho=\sqrt{\omega^{2}-m^{2} r}$ whose solutions are non-relativistic Coulomb Wave functions. $L_{l}(\varrho)$ and $K_{l}(\varrho)$ whose definitions are given in [19],

$$
R=A L_{l}(\varrho)+B K_{l}(\varrho)
$$

for large $x$ we have:

$$
\begin{aligned}
& L_{l}(\varrho) \rightarrow \frac{1}{\varrho} \sin \left(\varrho-\frac{l \pi}{2}+\eta_{i}-\alpha \log 2 \varrho\right) \\
& K_{l}(\varrho) \rightarrow \frac{1}{\varrho} \cos \left(\varrho-\frac{l \pi}{2}+\eta_{i}-\alpha \log 2 \varrho\right)
\end{aligned}
$$

with

$$
\eta_{l}=\arg \Gamma(l+1+i \alpha) .
$$

By matching these solutions to those in (4.30) we obtain:

$$
\begin{aligned}
\frac{B}{A}= & \frac{[l !]^{4}}{[2 l !]^{2}} \frac{2^{2 l-1}}{[(2 l+1) !]^{2}}\left[\sqrt{\omega^{2}-m^{2}}\left(r_{+}-r_{-}\right)\right]^{2 l+1} \\
& \cdot \exp -\pi \alpha\left|\frac{\pi \alpha}{\sin h \pi \alpha}\right| \prod_{\substack{s=1 \\
s=l}}\left(s^{2}+\alpha^{2}\right)\left(1+\frac{4 F^{2}}{s^{2}}\right)-2 i F .
\end{aligned}
$$

The absorbtion coefficient we require is:

$$
A_{\alpha}=1-\left|\frac{A+i B}{A-i B}\right|^{2} \approx-4 \mathscr{I}_{m}\left(\frac{B}{A}\right) \quad \text { since }|F| \ll 1 .
$$

Thus we obtain

$$
\begin{aligned}
A_{\alpha}= & \frac{4 F 2^{2 l}}{[(2 l+1) !]^{2}} \frac{[l !]^{4}}{[2 l !]^{2}}\left\{\sqrt{\omega^{2}-m^{2}}\left(r_{+}-r_{-}\right)\right\}^{2 l+1} \\
& \cdot \exp -\pi \alpha\left|\frac{\pi \alpha}{\sin h \pi \alpha}\right| \prod_{s=1}^{s=l}\left(s^{2}+\alpha^{2}\right)\left(1+\frac{4 F^{2}}{s^{2}}\right) \operatorname{sign}(\omega) .
\end{aligned}
$$

The absorbtion cross section $\sigma$ is given by

$$
\sigma=\sum_{l=0}^{l=0} \frac{A_{l} \pi}{\left(\omega^{2}-m^{2}\right)} .
$$

If we include just $l=0$ ("s wave") term we obtain:

$$
\sigma=4 \pi r_{+}^{2}\left(\omega+\frac{e Q}{r_{+}}\right) \frac{1}{\sqrt{\omega^{2}-m^{2}}} e^{-\pi \alpha}\left|\frac{\pi \alpha}{\sin h \pi \alpha}\right| \operatorname{sign}(\omega) .
$$


To obtain the relevant formula for rotating charged black holes it is sufficient to replace $F$ by $\frac{1}{2 \kappa}\left(\omega+e \Phi_{H}-n \Omega\right)$ where $n$ is the angular momentum quantum number, $\Omega$ is the angular velocity of the black hole and $\Phi_{H}$ the electrostatic of the hole.

Note that as $\omega \rightarrow m$ the absorbtion coeff. $A_{i}$ tends to a finite limit althoug the cross section diverges. The latter fact is typical of Coulomb cross sections and in practice causes no problem since one considers a range of energies for the incident particles. This is a rather different behaviour from the $m=0, e Q=0$ case in which $\sigma \rightarrow$ the area of the event horizon as the wavelengths tend to infinity, as pointed out by Starobinsky in the rotating case. This shows that the belief commonly expresses that particles whose de Broeglie or Compton wavelength is large compared with the Schwarzchild radius cannot "fit" inside a black hole is a mistaken one. If the charge is of the same sign as the black hole - i.e. $\omega<0$ then $\alpha$ can be $+e$ and a strong Coulomb barrier reduces the absorbtion cross section by an exponential factor - this is familiar from usual atomic situation (15).

To quantify this effect we introduce what we shall call a "Coulomb barrier penetration factor" $f(\alpha)$ defined by

$$
f(\alpha)=\exp -\pi \alpha|\operatorname{cosech} \pi \alpha|
$$

which obeys $1 \leqq f \leqq 2$ for positive $\omega$ and also for negative $\omega$ if $M m>e Q$. If however $M m<e Q$ then $f(\alpha)$ is bounded above by 2 but falls sharply to zero as $\omega \rightarrow-m$. This means that the superradiant coefficients will be rather small. It is important to note that it is this second case which will prevail in practice since any charged hole whose mass is less than $e^{2} m^{-1}$ must have $M m<e Q$ as is clear from Fig. 1.

We shall not proceed further and compute detailed formulae for the charge loss, but merely remark that even though the cross section $A^{(-)}$for modes which tend to discharge the hole is diminished compared with the charging modes, $A^{(+)}$, crude estimates indfcate that the net effect is to discharge the hole rapidly.

\section{Conclusion}

We have shown how a charged black hole can lose its charge by spontaneously emitting particles, assuming that the back reaction of the quantum fields on the external electromagnetic and gravitational field is negligible. For large black holes this is probably a reasonable approximation - for holes smaller compared with $\frac{1}{m}$ it is probably rather poor. We have seen that the external field will produce a polarization of the vacuum which will produce a modification of the external field. By analogy with the calculations for electrons one expects this effect to be important at distances smaller compared with $\frac{1}{\mathrm{~m}}$. Unfortunately any calculation of the effect is likely to prove rather difficult since the functions $p_{i}$ and $n_{i}$ cannot be calculated exactly. An alternative approach would be to construct a Feynman propagator for the fields but this is also not easy. 
Another, but less serious difficulty is that we have treated only spinless particles. For spin half fermions the theory is similar but more complicated due to the extra spin degrees of freedom. Also the classification of particle and antiparticle states is rather different, since the natural norm one takes is positive definite. The method one then adopts is to classify the states using the sign of the "energy"

$$
\int \bar{\psi} \gamma_{\mu} K^{\alpha} \mathscr{D}_{\alpha} \psi d \Sigma^{\mu}
$$

Most of the general analysis can be done as before although the detailed form of the scattering coefficients will of course be different. For higher spins the situation is less clear. There do not seem to be any obstacles with spin one particles but for spins greater than one seem to be a variety of difficulties $[20,21]$.

The implications for the detectability of small black holes are rather dissapointing since they will have evaporated by now if they are too small and those that are not must be rather scarce. If their total density of black holes of mass $10^{17} \mathrm{~g}$ say is to be less than the so called "magic density" then fewer than one would be expected to strike the earth in $10^{9}$ years.

Acknowledgement. I should like to thank S. W. Hawking for helpful discussions.

\section{References}

1. Hawking, S. W.: Mon. Nat. Roy. Astro. Soc. 152, 75-78 (1971)

2. Bardeen, J. M., Carter, B., Hawking, S. W.: Commun. math. Phys. 31, 161-170 (1973)

3. Ruffini, R., Denardo, G.: Phys. Letters. 45 B, 259-262 (1973)

4. Penrose, R.: Riv. Nuovo. Cim. 1, 252-275 (1969)

5. Bjorken, J. D., Drell, S. D.: Relativistic quantum mechanics. New York: McGraw Hill 1965

6. Schwinger, J.S.: Phys. Rev. 82, $664-679$ (1951)

7. Gibbons, G.W., Hawking, S. W.: In: De Witt-Morette,C. (Ed.): Gravitational radiation and gravitational collapse. Reidel 1974

8. Hawking, S. W.: Nature 248, 30 (1974)

9. Hawking, S. W.: Commun. Math. Phys. (to appear)

10. Starobinsky, A. A.: Zh.E.T.F. 64, 48, Eng. trans. J.E.T.P. 37, 28 (1973)

11. Unruh, W.: Phys. Rev. D. 10, 3195-3205 (1974)

12. Dyson, F. J.: Article in: Salam, A., Wigner, E. (Eds.): Aspects of quantum theory. C.U.P. 1973

13. Uehling, E. A.: Phys. Rev. 48, 55 (1935)

14. Weichmann, E.H., Kroll, N. M.: Phys. Rev. 101, 843 (1956)

15. Fulling, S. A.: Phys. Rev. D 7, 2850 (1973)

16. Snyder, H.S., Weinberg, J: Phys. Rev. 57, 307 (1940)

17. Landau,L., Lifshitz,E. M.: Quantum mechanics. London: Pergamon 1959

18. Churilov, S. M., Starobinsky, A.A.: Zh.E.T.F. 65, 3 (1973)

19. Mott, N.F., Massey, H.: The theory of atomic collisions, 2nd ed. London: O.U.P.

20. Buchdahl, H. A.: Nuovo Cimento 25, 486 (1962)

21. Wightman, A.S.: Troubles in the external field problem for invariant wave equations. New York: Gordan and Breach 1971 see also the article by Wightman in Ref. [12]

Communicated by J. Ehlers 